\title{
COMENTARIO A UN SONETO DE GONGORA*
}

\section{Francisco Domínguez Matito}

De pura honestidad templo sagrado, cuyo bello cimiento y gentil muro de blanco nácar y alabastro duro fue por divina mano fabricado; pequeña puerta de coral preciado, claras lumbreras de mirar seguro, que a la esmeralda final el verde puro habéis para viriles usurpado;

soberbio techo, cuyas cimbrias de oro al claro sol, en cuanto en torno gira, ornan de luz, coronan de belleza;

ídolo bello, a quien humilde adoro, oye piadoso al que por ti suspira, tus himnos canta, y tus virtudes reza.

Si nos fiamos de la cronología del célebre manuscrito Chacón, este soneto lo escribió Góngora en 1582. Es, por lo tanto, de los más tempranos que conservamos. En la edición de los sonetos gongorinos de Biruté Ciplijauskaité, encabeza la serie de sonetos amorosos, con un pequeño comentario de fuentes'; y también ha merecido unas palabras de Dámaso Alonso, al tiempo que su selección por el gran gongorista ${ }^{2}$.

* Entregado el manuscrito a la revista, leo el comentario que hace a nuestro soneto Andrés Romarís Pais en Manojuelo de Estudios Literarios ofrecidos a José Manuel Blecua Teijeiro por los profesores de Enseñanza Media, Madrid, M.E.C., 1983, pág. 103-117. No he podido por eso recoger sus conclusiones. Con dicho comentario tiene el nuestro algunas coincidencias de interpretación; no obstante, se trata de dos comentarios distintos.

1. Luis de Góngora: Sonetos completos, Edición, introducción y notas de Biruté Ciplijauskaité, Madrid, Castalia, 1969, p. 111.

2. Vid. Dámaso Alonso: Góngora y el "Polifemo", II, Madrid, Gredos, 1974, pp. 131-132. 


\section{FRANCISCO DOMINGUEZ MATITO}

En el año 1582 Góngora tenía veintiún años. Hacía dos que había terminado sus estudios en Salamanca, y se encontraría probablemente en Córdoba, su Córdoba cantada en versos inolvidables. Dos años hacía igualmente que había aparecido su primera obra impresa: una canción al frente de la traducción al castellano del poema portugués Os Lusiadas de Camoens, por Luis de Tapia. En 1582 la fama de nuestro poeta iba creciendo: dos años después, un soneto suyo figuraba en los preliminares de $L a$ Austriada de Juan Rufo, y en 1585 Cervantes lo elogia merecidamente en el Canto de Caliope:
En don Luis de Góngora os ofrezco
un vivo raro ingenio sin segundo;
con sus obras me alegro y enriquezco
no sólo yo, mas todo el ancho mundo ${ }^{3}$.

Así, pues, Góngora, cuando escribió este soneto, comenzaba a convertirse en el más famoso poeta español. Estaba atravesando una época de tranquilidad en su vida, ignorante de todas las preocupaciones que más tarde le amargarían -ipobre!- durante su estancia en la Corte. Con veintiún años redactó esta composición cultista, línea estilística que simultaneará a lo largo de su vida con la vena popular humorística e irónica de sus deliciosas letrillas. El joven poeta de 1582 aún no podía hacer otra cosa que sumergirse en la corriente culta del petrarquismo que llenaba la segunda mitad del siglo XVI. Será a fuerza de años cuando logre desprenderse de los modelos italianos para crear su propio lenguaje, pero en estos momentos la corriente le arrastraba y a ella tuvo que pagar su tributo; de ahí que su primeriza poesía, como el ejemplo que tenemos delante, ceda a la utilización de una serie de fórmulas comunes que le confieren un tono impersonal, frío, demasiado artificioso. El alma singular del poeta, todavía, se nos escapa de entre las manos. Después de leer los dos cuartetos y el primer terceto de nuestro poema, absolutamente descriptivos, las referencias personales del último terceto no nos resultan suficientes ni emocionantes: todo parece desenvolverse en un juego retórico, en un puro formalismo. Es difícil, por eso, suscribir la opinión de Dámaso Alonso cuando califica este soneto como "uno de los más emocionados y tiernos de nuestro poeta"4. A la imaginería de Petrarca, cuya fuente es la naturaleza en su estado perfecto y pleno de belleza, pertenece el léxico empleado aquí para referirse a los miembros de la amada: nácar, alabastro, coral, esmeralda, sol, luz. Vocabulario preciosista, todo de color. Y típico de la tradición petrarquista del Renacimiento es el intento de reflejar la naturaleza en todo, resaltando lo arquetípico, la cualidad fundamental

3. Cervantes: La Galatea, II, Edición, introducción y notas de J.B. Avalle-Arce, Madrid, España-Calpe, Clásicos Castellanos, 1968, p. 210.

4. Vid. Dámaso Alonso: op. cit., p. 132. 


\section{COMENTARIO A UN SONETO DE GONGORA}

de las cosas, lo más estimado en el ser. A esta concepción responden los epítetos sagrado (templo), blanco (nácar), duro (alabastro), preciado (coral), claras (lumbreras), fina (esmeralda), claro (sol), con total evidencia. Estos convencionalismos ahogaron en el joven don Luis la ternura, la pasión, quizá esperables de su edad temprana ${ }^{5}$.

Se han señalado para este soneto varias fuentes: Minturno, "In si bel tempio di memorie adorno"; la Egloga I de Garcilaso, “DDó la columna que el dorado techo / con presunción graciosa sostenía?"; Petrarca, "Tacer non posso" . Es claro que Góngora estaba frente a una tradición que conocía muy bien, lo que excluye a cada texto en particular como inspirador directo del poema que comentamos. El tema-motivo convencional de la comparación de los miembros de la amada con metales o elementos preciosos, se encontraba por todas partes en la poesía inmediatamente anterior a Góngora. Petrarca pintaba así a su Laura:

L'atto d'ogni gentil pietate adorno, e 'I dolce amaro lamentar ch' i' udiva, facean dubbiar se mortal donna o diva fosse che 'l ciel rasserenava intorno.

La testa òr fino, e calda neve il volto, ebeno i cigli, e gli occhi eran due stelle, onde Amor l'arco non tendeva in fallo;

perle, e ròse vermiglie, ove l'accolto dolor formava ardenti voci e belle; fiamma i sospir, le lagrime cristallo ${ }^{7}$.

5. Vid. F. Lázaro Carreter: "Para una etopeya de Góngora", en Estilo Barroco y Personalidad Creadora, Madrid, Cátedra, 1974, pp. 129-147.

6. Vid. J.G. Fucilla: Estudios sobre el petrarquismo en España, Madrid, C.S.I.C., 1960, pp. 252-257.

7. El gesto, de toda gentil piedad adornado, y el dulce amargo quejarse que yo oía, hacían dudar si era mujer mortal o divina la que hacía serenarse el cielo alrededor.

Oro fino la cabeza, y cálida nieve el rostro, ébano las cejas y los ojos dos estrellas, desde las cuales Amor no tendía su arco en vano; perlas, y rosas bermejas, el sitio en que recogido el dolor formaba voces ardientes y bellas; llama los suspiros, las lágrimas cristal.

Francesco Petrarca: Obra completa en poesía. El Cancionero, Tomo I, Edición Bilingüe, Barcelona, Ediciones 29, 1976, pp. 286-287. 
Pronto encontramos el tópico en Garcilaso, que pregunta en la estancia 20 de la Égloga I:

\author{
¿Dó están agora aquellos claros ojos \\ que llevaban tras sí, como colgada, \\ mi alma, doquier que ellos se volvían? \\ ¿Dó está la blanca mano delicada, \\ llena de vencimientos y despojos \\ que de mí mis sentidos l'ofrecían? \\ Los cabellos que vían \\ con gran desprecio al oro \\ como a menor tesoro \\ ¿adónde están, adónde el blanco pecho? \\ ¿Dó la columna que'l dorado techo \\ con proporción graciosa sostenía? \\ Aquesto toda agora ya s'encierra, \\ por desventura mía, \\ en la escura, desierta y dura tierra ${ }^{8}$.
}

Como se ve, es el mismo lugar común descriptivo renacentista, al que se une en singular conjunción el viejo tema del ubi sunt medieval. El ideal femenino renacentista de cabellos rubios, ojos verdes, piel blanquísima, fue constante motivo de inspiración para los poetas. Lo hallamos en el famoso soneto XXIII del propio Garcilaso En tanto que de rosa y d'azucena, en su Canción $I V$, etc., y en versos muy conocidos de Gutierre de Cetina, Fernando de Herrera...

Ahora bien, la estancia 20 de la Egloga I garcilasiana, nos sirve no sólo para la constatación de la tradición del motivo que Góngora utiliza en nuestro soneto, sino también para contrastar dos sensibilidades, dos estilos. En efecto, resulta interesante observar cómo Garcilaso y Góngora proceden en la descripción con un orden distinto. Aquel comienza evocando los ojos, baja a las manos, sube después hasta los cabellos, desciende nuevamente al pecho, y se refiere por fin al cuerpo para terminar otra vez dirigiendo su mirada a la cabeza. Se trata de un movimiento desordenado, caótico, atropellado. Pero la descripción de Góngora se sujeta a una matemática perfecta, a un ordenamiento sereno y progresivo: empieza por las piernas para ir ascendiendo gradualmente hasta llegar a la cabeza. Me parece claro que en el desorden garcilasiano está expresada la propia naturaleza ilógica y apasionada del sentimiento, mientras que la simétrica mirada gongorina es fruto de una racionalísima artesanía poética, que oculta la vibración espiritual. El poeta toledano llora en versos emocionantes la pérdida de una amada real;

8. Garcilaso de la Vega: Poesías Castellanas Completas, Edición, introducción y notas de Elías L. Rivers, Madrid, Castalia, 1972, p. 129. 
el cordobés suplica a un "ídolo bello" del que no atisbamos la corporeidad. En mi opinión, estas dos composiciones representan el principio y el final de un estilo, la fresca autenticidad de Garcilaso y la impersonalidad del primer Góngora culto.

Rastrear la presencia del tópico comparativo-preciosista en la obra de Góngora, es hacer la historia de su evolución poética desde los modelos italianos hasta la adquisición de su peculiar estilo barroco. Ya en 1582 vuelve sobre él en el magnífico soneto Mientras por competir con tu cabello. En éste la descripción se inicia con el cabello, oro bruñido, continúa por la blanca frente, baja después a los labios, para concluir con el gentil cuello. El discurso de la mirada es, como en nuestro caso, rigurosamente metódico; las partes guardan en la estructura del soneto la misma posición que ocupan en la realidad, identificándose así orden natural con orden poético. En 1583, y también con el tema del carpe diem, escribe el soneto Ilustre y hermosísima María, de similar repertorio amoroso. Un soneto de 1603 está escrito bajo la inspiración de "tus ojos, dulcísima señora", y el poeta en el último terceto ve la frente de la dama coronada de rayos, igual que en nuestro caso. En una composición de 1607 dedicada a Doña Brianda de la Cerda, vuelven a aparecer los cabellos de Clori que oscurecen al mismo sol. Clori tiene unos "divinos ojos" que "dan luz al mundo". Curiosamente, el primer cuarteto de este poema lo repite íntegro en otro de 1620 en el que sólo cambia el nombre de la dama: Peinaba al sol Belisa sus cabellos.

El recurso de comparar los miembros de la mujer con perlas y joyas, omnipresente en los primeros y segundos pasos de Góngora, lo encontramos todavía en la Fábula de Polifemo y Galatea: la bella ninfa es de color blanco, rosado, posee unos lucientes ojos, orejas de nácar, frente de perla. Hay 31 años de diferencia entre Galatea y el ídolo de 1582 y, sin embargo, Góngora mantiene el vocabulario, la fórmula, el simétrico rigor descriptivo. Ahora bien, cotejar los dos textos pone en evidencia lo que es un "estilo en movimiento", porque la utilización que el Góngora maduro del Polifemo hace de los procedimientos petrarquistas es muy distinta a la del soneto juvenil. Ahora, los elementos están al servicio de otra concepción del mundo, no se entienden aislados sino en el entrecruzamiento intensificativo de los contrastes barrocos ${ }^{9}$. La belleza de Galatea se integra en un conjunto del que depende su funcionalidad y su sentido; pero el "ídolo bello" del soneto, como los otros ejemplos primerizos, forman piezas singulares.

El punto final en el tratamiento del tópico se encuentra en la Fábula de Píramo y Tisbe, de 1618, en la que el escritor, definitivamente poseedor de un nuevo sistema expresivo, se burla con orgulloso distanciamiento de los

9. Vid. Dámaso Alonso: Poesía española. Ensayo de métodos y límites estilisticos, Madrid, Gredos, 1971, pp. 351-392. 


\section{FRANCISCO DOMINGUEZ MATITO}

procedimientos del período anterior ${ }^{10}$. En un poema en el que lo culto se mezcla con lo vulgar, lo cómico con lo serio, la belleza de Tisbe participa de ambos extremos: su tez es luciente cristal; su boca, un rubí; sus dientes son veinte perlas; pero su nariz se parece a un blanco almendruco, sus pechos son pechugas o pomos no maduros cuando no sólo "relieves ocultos", y su cuerpo es un "etcétera" de mármol". En esta onda se produce la coincidencia entre Góngora y Cervantes, que hace hablar así a Don Quijote de la belleza de Dulcinea:

...su hermosura, sobrehumana, pues en ella se vienen a hacer verdaderos todos los imposibles y quiméricos atributos de belleza que los poetas dan a sus damas: que sus cabellos son oro, su frente campos elíseos, sus cejas arcos del cielo, sus ojos soles, sus mejillas rosas, sus labios corales, perlas sus dientes, alabastro su cuello, mármol su pecho, marfil sus manos, su blancura nieve, y las partes que a la vista humana encubrió la honestidad son tales, según yo pienso y entiendo, que sólo la discreta consideración puede encarecerlas y no compararlas ${ }^{12}$.

La fina ironía cervantina se expresa en la soberbia acumulación de los tópicos comparativos y en la descripción minuciosamente ordenada del cuerpo de Dulcinea, recorrido de arriba abajo.

Puesto que Don Luis de Góngora pretendió siempre crear un sistema poético difícil, un lenguaje de exquisita y condensada poeticidad, resulta útil desde el punto de vista metodológico la prosificación de sus poemas, para así comprender en cada caso el secreto de sus significaciones y efectos estéticos. Sin embargo, conviene advertir que el método de análisis que voy a emplear no aspira a ser una aplicación rigurosa de la estilística estructural, por más que esté relacionado con ella ${ }^{13}$. Se trata nada más que de examinar las desviaciones de la lengua poética gongorina respecto de la lengua normal, para medir su intensidad y cantidad. Por otra parte, si el análisis de estos dos caracteres es pertinente en cualquier poema, parece especialmente importante en la poesía de Góngora, cuya evolución está vinculada en gran medida con el progresivo uso que de ellos se hace.

10. Vid. F. Lázaro Carreter: "Situación de la 'Fábula de Píramo y Tisbe', de Góngora", en op. cit., pp. 45-68.

11. Luis de Góngora y Argote: Obras completas, Recopilación, prólogo y notas de J. Millé y Giménez y I. Millé y Giménez, Madrid, Aguilar, 1972, pp. 202-214.

12. Miguel de Cervantes: El Ingenioso Hidalgo Don Quijote de la Mancha, I, Edición, introducción y notas de Luis Andrés Murillo, Madrid, Castalia, 1978, p. 176.

13. Vid. Jean Cohen: Estructura del lenguaje poético, Madrid, Gredos, 1973. 
Mi ensayo de prosificación va a constar de dos fases. He aquí la primera:

Templo de honestidad cuyo cimiento y muro fue fabricado de nácar y alabastro por la mano de Dios; puerta pequeña de coral, lumbreras penetrantes que habéis quitado el verde a la esmeralda para hacer viriles; impresionante techo cuyas cimbrias amarillas iluminan al sol cuando gira alrededor de ellas; ídolo a quien adoro, oye con piedad al que suspira por ti, al que canta tus excelencias y proclama tus virtudes.

Esta prosificación conserva algunos rasgos poéticos ya comentados, de tipo léxico; otros serán reducidos en la segunda fase. Por obviedad tanto como por razones de espacio, prescindiré en el análisis de los resultados de la prosificación de pormenores, para destacar los fenómenos que considero más significativos.

En primer lugar, es evidente que los efectos poéticos pertenecientes al plano del significante, que definen de modo sustancial el estilo de Góngora, han quedado quebrados. Se ha perdido el ritmo y la medida, manifiestos en el soneto por los catorce perfectos endecasílabos, con el último acento localizado siempre en la penúltima sílaba métrica, la décima, de lo que resulta un axis rítmico isopolar. Asimismo, la situación del axis en unidad cuantitativa par, da signo yámbico a todo el ritmo de las estrofas. La posición acentual más general es $2^{\mathrm{a}}-4^{\mathrm{a}}-8^{\mathrm{a}}-10^{\mathrm{a}}$, las posiciones en $2^{\mathrm{a}}-6^{\mathrm{a}}-8^{\mathrm{a}}-10^{\mathrm{a}}$ y en $4^{\mathrm{a}}-8^{\mathrm{a}}-10^{\mathrm{a}}$ son menos frecuentes. Existen varios acentos extrarrítmicos: en el segundo v. hay uno en $3^{\mathrm{a}}$ (bello); en el v. cuarto en $1^{\mathrm{a}}($ fue $)$; en el v. sexto en $1^{\mathrm{a}}$ ( claras); en el v. once en $1^{\mathrm{a}}$ (ornan); en el v. doce en $1^{\mathrm{a}}$ (ídolo); en el v. trece en $1^{a}$ (oye). Seis acentos extrarrítmicos en total. Aparece también un acento antirrítmico en el primer verso en $7^{\text {a }}$ (templo), y otro antiestrófico en la sílaba $9^{\mathrm{a}}$ del segundo verso (gentil). Tal distribución acentual equilibrada y simétrica nos permite calificar el ritmo del soneto de intensivamente ponderado. Ahora bien, el importante número de acentos no rítmicos nos obliga a una interpretación métrico-estilística. El antirrítmico de templo viene a reforzar la relevancia impresivo-expresiva de esta palabra. El extrarrítmico de bello y el antiestrófico de gentil, colocados ambos en adjetivos, realzan la misión calificativa de éstos, adjuntos de dos palabras semánticamente relacionadas: cimiento y muro. El extrarrítmico del cuarto verso colocado sobre la forma temporal fue subraya con intensidad el tiempo pasado. En el primer terceto, el extrarrítmico sobre el verbo ornan acentúa la rapidez de la acción señalada en el verso anterior. En fin, el apresuramiento del poeta en el último terceto, el tono suplicante, están intensificados por los acentos extrarrítmicos de ídolo y oye. Sintetizando, los acentos no concordes con la estructura yámbica de los versos, y por tanto realzadores, se hallan sobre palabras que constituyen el esqueleto semántico del soneto: 


\section{FRANCISCO DOMINGUEZ MATITO}

$\begin{array}{ll}\text { templo } & \text { (sagrado) } \\ \text { bello } & \text { (cimiento) } \\ \text { gentil } & \text { (muro) } \\ \text { fue } & \text { (fabricado) } \\ \text { claras } & \text { (lumbreras) } \\ \text { ornan } & \text { (de luz) } \\ \text { idolo } & \text { (bello) } \\ \text { oye } & \text { (piadoso) }\end{array}$

Nada de esto, naturalmente, puede concebirse en una prosificación, la cual desconoce también el efecto estético de la rima cuyo esquema en nuestro soneto es ABBA ABBA CDE CDE.

Manteniéndonos dentro del terreno de la expresión, conviene resaltar la desaparición en la versión a prosa de la musicalidad de los versos, conseguida por la aliteración de los fonemas consonánticos armónicos l-r. Al propio tiempo se desvanece la sensación de luminosidad que transmite la multitudinaria presencia de las vocales a-e-i en aliteración, sensación que, a mi juicio, encierra el misterio de este soneto.

Examinemos ahora las desviaciones que la lengua poética gongorina presenta en cuanto al orden de las palabras. Hay en el poema un equilibrio casi perfecto entre la anteposición y la posposición del adjetivo. De los diecisiete que acompañan al sustantivo, 9 se anteponen, 8 se posponen ${ }^{14}$ :

$\begin{array}{llll}\text { v. } 1 & \text { pura honestidad } & \text { v. } 1 & \text { templo sagrado } \\ \text { v. } 2 & \text { bello cimiento } & \text { v. } 3 & \text { alabastro duro } \\ \text { v. } 2 & \text { gentil muro } & \text { v. } 5 & \text { coral preciado } \\ \text { v. } 3 & \text { blanco } \text { nácar } & \text { v. } 6 & \text { mirar seguro } \\ \text { v. } 4 & \text { divina } \text { mano } & \text { v. } 7 & \text { esmeralda fina } \\ \text { v. } 5 & \text { pequeña puerta } & \text { v. } 7 & \text { verde puro } \\ \text { v. } 6 & \text { claras lumbreras } & \text { v. } 9 & \text { cimbrias de oro } \\ \text { v. } 9 & \text { soberbio techo } & \text { v. } 12 & \text { ídolo bello } \\ \text { v. } 10 \text { claro } \text { sol } & & \end{array}$

Que el poeta ha concedido aquí gran importancia a la adjetivación, y que la anteposición-posposición en equilibrio alternativo logra un efecto estético deliberadamente buscado, se comprueba por la evidencia de cinco quiasmos:

v. 1 De pura honestidad templo sagrado

v. 3 de blanco nácar y alabastro duro

v. 5 pequeña puerta de coral preciado

v. 6 claras lumbreras de mirar seguro

v. 9 soberbio techo cuyas cimbrias de oro

14. A estos efectos he considerado el complemento "de oro" del verso 9 como adjetivo. 


\section{COMENTARIO A UN SONETO DE GONGORA}

Los adjetivos antepuestos quedan realzados por su posición en la cadena hablada; los pospuestos, por su situación al final del verso, toman los factores expresivos de la rima y del axis rítmico. Y con el quiasmo repetido la estructura del soneto adquiere una solidez arquitectónica.

En este mismo plano, no puede dejar de advertirse la presencia del hipérbaton, tan característico del gongorismo, que ayuda a reforzar el valor semántico de algunos elementos. Eso ocurre en el primer verso, cuya belleza deriva sin duda de su disposición hiperbática que hace aproximarse a dos acentos; en la colocación de los verbos adoro, suspira, canta y reza al final del período, detrás de los complementos, los que les hace coincidir con las cumbres rítmicas versales.

En el nivel predicativo, la prosificación revela también repetidas desviaciones en el lenguaje poético de nuestro soneto. Como se sabe, el epíteto tiene una misión determinativa y por ello debe aplicarse sólo a parte del nombre. El epíteto se convierte en "anormal" cuando no conviene a ninguna parte del nombre o cuando conviene a todas, en cuyo caso resulta ocioso, redundante o no pertinente. La utilización por los poetas de adjetivos en este sentido de anormalidad, aplicando a la cosas propiedades que no les son propias o les pertenecen por esencia, trasluce su singular visión del mundo, la impresión que el universo hace en su sensibilidad, la afectividad que derraman sobre los seres. El análisis de la adjetivación en un poeta es, pues, un rico manantial para la comprensión de su psicología y de su estilo. En el texto que comentamos son redundantes pura (honestidad), sagrado (templo), blanco (nácar), duro (alabastro), preciado (coral), claras (lumbreras), claro (sol). Siempre respecto al lenguaje denotativo de la prosa, resultan inapropiados bello (cimiento), gentil (muro), soberbio (techo). Estos adjetivos son así estilemas semánticamente innecesarios, pero, una vez más, rasgos renacentistas en tanto que reflejan la perfección de la naturaleza.

Estilemas renacentistas también, aunque de vieja historia, que recoge Góngora y utilizará amplísimamente a través de su obra como rasgo característico, son los que Dámaso Alonso ha estudiado tan repetidas veces: las bimembraciones y correlaciones ${ }^{15}$. En los versos 2 y 3 encontramos casos de bimembración

$\begin{array}{lll}\frac{1}{\text { bello cimiento }} & \text { y } & \frac{2}{\text { gentil muro }} \\ \frac{1}{\text { blanco nácar }} & \text { y } & \frac{2}{\text { alabastro duro }}\end{array}$

y una correlación, ya que se trata de dos pluralidades relacionadas: nácarcimiento, alabastro-muro. Otro fenómeno de correlación en pluralidades lo tenemos en el primer terceto:

15. Vid. Dámaso Alonso y Carlos Bousoño: Seis calas en la expresión literaria española (Prosa, Poesía, Teatro), Madrid, Gredos, 1970, pp. 23-41, 45-74 y 77-107. 


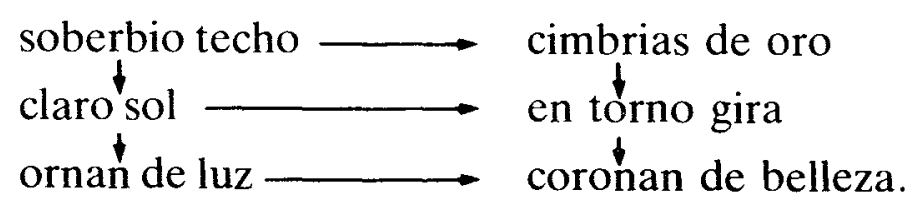

Es, desde luego, una correlación imperfecta porque la simetría de los elementos está oscurecida por las funciones sintácticas, pero el reflexivo uso del hipérbaton intenta aquí un tipo de referencias que Góngora perfeccionó más tarde. Parece perceptible aquí la relación -no del todo explícito- entre 'altura-sol-luz' y 'cabellos-vuelta-corona'. El verso 14 nos proporciona un último caso de bimembración:

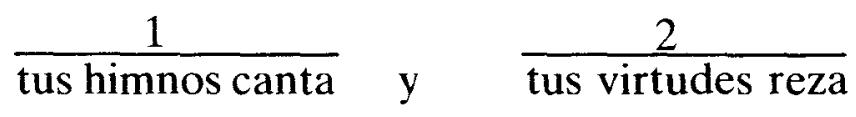

Con todo lo dicho ha quedado planteada la desviación de la lengua poética gongorina respecto de la norma lingüística usual, primera fase del procedimiento de la prosificación. La segunda fase de este proceso consiste en reducir la desviación para hallar la "gramática" del soneto, la inteligibilidad definitiva del mensaje poético. La poesía, en general y en cada caso concreto, es un universo en el que un haz de relaciones de significado determina el término de referencia de cada significante. Mediante este estatuto gramatical distinto al de la prosa, los diversos elementos que en la lengua general estarían desprovistos de funcionalidad, toman sentido en la poesía.

En la segunda vuelta del ensayo de prosificación quedan desvelados los términos reales de los sustantivos metafóricos, clave organizadora de la poeticidad de este texto.

Veamos:

Mujer amada, que eres como un templo de honestidad cuyos cimientos son tus pies y sus paredes tu cuerpo; cuya puerta es tu boca de labios rojos como el coral; cuyas lumbreras son tus ojos verdes; cuyo techo es tu cabeza cubierta de deslumbrante cabello rubio; escucha con clemencia al que humildemente te adora como a un dios.

Ahora, entendido que con los elementos arquitectónicos el poeta se refiere a las distintas partes del cuerpo femenino, que se compara con un templo, la predicación de los adjetivos adquiere toda su funcionalidad expresiva, se vuelve pertinente y pierde su carácter prosopopéyico. La clave significativa, como decía, de este soneto alegórico reside en el plano real de los sustantivos. A lo largo de los once primeros versos, el poeta, extasiado, describe el cuerpo de la amada. En el último terceto aparece el amante. Revestida la dama con atributos divinos, el enamorado se sitúa frente a ella con una actitud religiosa. La multiplicidad de verbos (adoro, oye, suspira, canta, reza) 


\section{COMENTARIO A UN SONETO DE GONGORA}

y los dos adjetivos (humilde, piadoso) matizan bien el sentimiento de pequeñez desde el que se implora compasión a un dios, al que se anhela y se le adora proclamando sus maravillas. He aquí, por tanto, otro ejemplo culminante del espíritu secular del poeta renacentista.

En resumen. Nuestro soneto representa un ejemplo del Góngora inicial, aún rendido al más puro estilo petrarquista que inunda la poesía amorosa pagana del siglo XVI. Todos los convencionalismos temáticos y estilísticos se dan cita en este poema, exponente de la línea cultista del autor. Sin embargo, no dejan de percibirse unas tendencias que van a caracterizar el inconfundible Góngora barroco: la acumulación de elementos retóricos, el léxico suntuario, colorista y sonoro, el uso de determinadas fórmulas estilísticas, la perfección estructural, la absoluta metaforización de la realidad, el ahogo de la libre expresión del sentimiento por la matemática de la forma.

Aunque en este soneto de 1582 Góngora no ha alcanzado todavía su propio lenguaje, ello no menoscaba su indudable calidad. El poeta ha pretendido comunicarnos su fascinación ante la acabada belleza de una mujer, y eso lo ha conseguido plenamente con la utilización de un procedimiento enaltecedor, con el acumulativo léxico preciosista y de color, de los que derivan poderosas sensaciones de belleza, blancura y perfección. Intención y ejecución, como decía Cernuda ${ }^{16}$, encuentran en este poema una exacta coincidencia. Sólo que la sensibilidad moderna preferiría tal vez contemplar un poco menos al ídolo, y sentir un poco más al hombre. En definitiva, el soneto gongorino al que nos hemos acercado no hace más que apuntar al gran gigante de la forma de la poesía española del siglo XVII. 
